\title{
Ground Based Laser Torque Effects on Both Passive and Active Control for LEO CubeSat
}

\author{
Thanaa E. Sharaf-Eldin ${ }^{1,2}$, Nabawia S. Khalifa ${ }^{2,3}$ \\ ${ }^{1}$ Electrical. Eng. Department, Faculty of Engineering, Alexandria University, Alexandria, Egypt \\ ${ }^{2}$ Basic Sciences Department, Deanship of Preparatory Year- Girls Branch, University of Hail (UOH), Hail, Saudi Arabia \\ ${ }^{3}$ Sun and Space Research Department, National Research Institute of Astronomy and Geophysics, Helwan, Egypt \\ Email address: \\ t.sharafeldeen@uoh.edu.sa (T. E. Sharaf-Eldin), asmaa_2000_2000@yahoo.com (N. S. Khalifa)
}

\section{To cite this article:}

Thanaa E. Sharaf-Eldin, N. S. Khalifa. Ground Based Laser Torque Effects on Both Passive and Active Control for LEO CubeSat. International Journal of Systems Science and Applied Mathematics. Vol. 2, No. 2, 2017, pp. 57-63. doi: 10.11648/j.ijssam.20170202.12

Received: March 15, 2017; Accepted: March 27, 2017; Published: April 13, 2017

\begin{abstract}
The objective of this work is to investigate the effects of laser torque on both passive and active control for LEO CubeSat. The investigation is based on the model of the Euler equations for rotational motion. A simple case of spacecraft attitude control problem is considered, in which passive control is performed by accounting only gravity gradient torque, and then active control technique is implemented using PD control and one momentum wheel. The system simulation is conducted using the package of Matlab 7.10-(R2010a). The simulated results show the effect of applying different magnitudes of ground based laser torque on the system stability for passive and active control. Having applied the ground based laser torque on passive and active control techniques respectively; the obtained results show that the laser torque possesses negative effects on the system performance for the current study. For passive control, the ground based laser torque has a significant effect where the roll and yaw angles' responses are diverged. However, with active control, the effect of laser torque is limited by a proper PD gains tuned.
\end{abstract}

Keywords: LEO Cubesat, Passive Control, Active Control, Euler Equations, PD Control, Ground Based Laser Torque

\section{Introduction}

Nowadays, CubeSats become the most popular spacecrafts due to their physical size and weight. They have relative simple hardware requirements for moderate attitude control, which results in an attractive short period of design, low energy and low cost. CubeSats' specifications are used to help universities and research institutes worldwide to carry out space science and exploration researches.

For extremely accurate satellite positioning and orientation, both satellite orbital and rotational motion must be modeled. The current work deals with satellite attitude control which plays a significant role to preserve the satellite in its designed attitude. Satellite attitude control systems are divided into two categories: passive and active control systems. Passive systems depend on the natural forces (i. e. gravity gradient, solar radiation pressure, aerodynamics and earth's magnetic field) for stabilization and control. These methods have been used due to their simplicity and low cost. Nevertheless, they can provide low pointing accuracies and limited control torques because of their dependability on the gravitational field and the geomagnetic field conditions.

Active control system consists of control elements and control algorithm. The control elements such as reaction wheels, thrusters, control moment gyroscopes or magnetic torquers are commonly used for spacecraft. However, the magnetic torquer is widely used as an actuator for geostationary satellites, small satellites and microsatellites. These high-tech devices interact with the Earth's magnetic field and create control torque, which can be adjusted to the required value. Combined with one or more reaction wheels, they provide all control needs to maintain the spacecraft's attitude [4] and [9] (Karla (2009) and Vincent (2010)). Unlike thrusters, magnetic torquers are light weighted, reliable, and energy-efficient. A further advantage over momentum wheels and gyroscopes is the absence of moving parts, which leads to a significantly higher reliability. However, it depends on Earth's magnetic field strength which 
results in highly nonlinear attitude control problem [8] and [9] (Samir (2009) and Vincent (2010)). For control algorithm, the conventional linear controllers; Proportional Derivative (PD), Proportional Integral (PI) and Proportional Integral Derivative (PID) are the commonly choices due to their design and simplicity. The main drawbacks of linear controllers are disregarding the uncertainty in satellite modeling and neglect nonlinear effects. The aforementioned factors lead to long settling time. On the other hand, the nonlinear control techniques include the effect of nonlinear dynamics. Variable structure control designs have attracted some researchers to implement them for attitude control of spacecraft. Particular attention has been given to the sliding mode control design, which has the advantage of reducing the system order to generate the control command. Moreover, the sliding mode presents the system robustness against to the external disturbances taking into consideration the model uncertainties [1] (E. Abdualhamitbilal et al.).

Recently, the possibility of using laser power for space applications became of great interest. There are several advantages of using lasers over other techniques of beamed power; the laser receiver is the solar array, so no new receiver technology is needed. Moreover, space laser beam suffers a less amount of atmospheric attenuation compared with the other beamed power applications. Also, it can be adjusted to be safe in use (i. e. no damage in the satellite surface) (Elsaftawy et al. (2007), El-saftawy and Makram (2004), Geoffery (1994) and Khalifa (2009)). With the intention of overcoming some disadvantages of the traditional attitude actuators, the issue of this work is to investigate the feasibility of using different ground based laser torques for passive and active attitude control of low Earth orbit CubeSats.

This paper is organized as follows: section "II" reviews the Euler equations of rotational motion, where the disturbing torques is that due to gravity gradient while the other disturbance will be ignored. The ground based laser torque of different magnitudes is worked out as control torque. Section III illustrates the system simulation and results for both passive and active control using the package of Matlab 7.10-(R2010a). Passive control will be studied first under the effect of gravity gradient only, and then in presence of both laser and gravity gradient torques. Active control technique based on PD controller is implemented for a momentum biased cubesat, which is supported by one reaction wheel. Then simulation is conducted to illustrate the effect of laser torque with different power range on response of yaw and roll angles. The conclusions and suggestions for future work are found in section IV.

\section{System Modeling}

\subsection{Reference Frames}

In this section, the three main reference frames are explained in the following subsections [4], [8] and [9] (Karla
(2009), Samir (2009) and Vincent (2010)):

\subsubsection{Earth Centred Earth Fixed (ECEF) Frame}

It is an Earth centred coordinate system which rotates relative to the Earth Centred. Its unit vectors are: $x$ which is extended to to the zero latitude and longitude point (i. e. the intersection of the Equator and the prime meridian passing through Greenwich, UK), $z$ is the earth spin axis which is pointing towards the celestial North Pole and $y$ completes the right- handed system.

\subsubsection{Earth Centred Inertial (ECI) Frame}

It is fixed with respect to inertial space and centered at the Earth's center of mass with unit vectors: $x_{N}$ directed towards the vernal equinox, $z_{N}$ directed towards the ecliptic pole and $y_{N}$ completes the right- handed system.

\subsubsection{The Body Fixed Frame}

It is centered at the center of mass of the satellite with unit vectors: $z_{b}$ is the pointing towards the Earth's center (Yaw axis), $y_{b}$ is directed normal to the satellite orbital plane (Pitch axis) and $x_{b}$ completes the right- handed system (Roll axis) As shown in figure 1.

It's worth noted that, the rotation between the body-fixed frame and ECI is considered to be the satellite attitude which is the focus in attitude control problems.

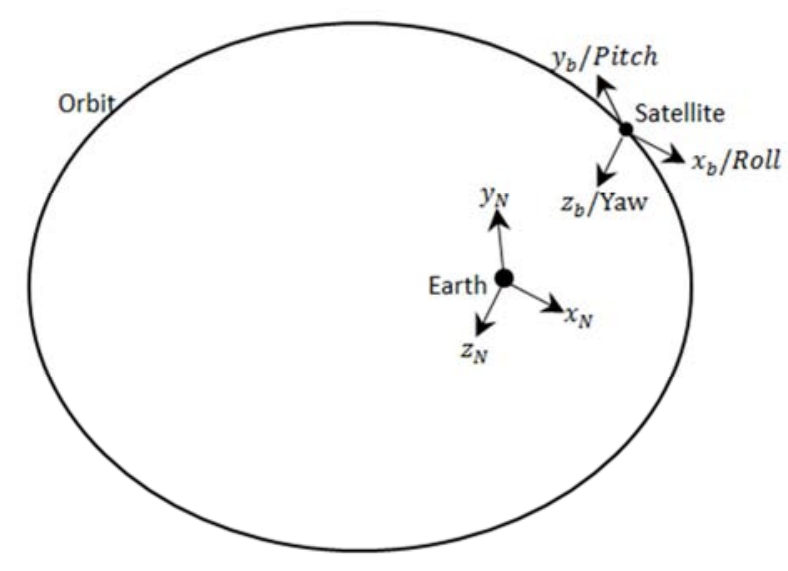

Figure 1. The body fixed frame.

\subsection{Satellite Rotational Dynamics}

\subsubsection{Equations of Motion}

For a momentum biased satellite in which the principle moments of inertias are aligned through the body fixed frame, the linear differential equations of motion are (Sidi, 1997; Wertz, 1978, Hsu et al., 1965):

$$
\begin{gathered}
T_{C x}+T_{d x}=I_{x} \ddot{\phi}+4 \omega_{o}^{2}\left(I_{y}-I_{z}\right) \phi+\omega_{o}\left(I_{y}-I_{z}-\right. \\
\left.I_{x}\right) \dot{\psi}+\dot{h}_{w x}-\omega_{o} h_{w z}-\dot{\psi} h_{w y o}-\omega_{o} \phi h_{w y o} \\
T_{C y}+T_{d y}=I_{y} \ddot{\theta}+3 \omega_{o}^{2}\left(I_{x}-I_{z}\right) \theta+\dot{h}_{w y}
\end{gathered}
$$

$T_{C z}+T_{d z}=I_{z} \ddot{\psi}+\omega_{o}\left(I_{z}+I_{x}-I_{y}\right) \dot{\phi}+\omega_{o}^{2}\left(I_{y}-I_{x}\right) \psi+$

$$
\dot{h}_{w z}+\omega_{o} h_{w x}+\dot{\phi} h_{w y o}-\psi \omega_{o} h_{w y o}
$$


where $T_{d x}, T_{d y}$ and $T_{d z}$ are the components of disturbing torques and $T_{c x}, T_{c y}$ and $T_{c z}$ are the components of control torques. The angles $\phi, \theta$ and $\psi$ are the Euler angles which describe the satellite rotations around its axes: $\phi$ represents rotation around $x, \theta$ represents rotation around $y$ and $\psi$ represents rotation around $z$. The term $\omega_{o}$ is the angular orbital velocity of the satellite which is called the orbital rate or frequency. $h_{w x}, h_{w y}$ and $h_{w z}$ are the angular momentum components of the momentum wheels in the body fixed frame. $I_{x}, I_{y}$ and $I_{z}$ are the components of moment of inertia tensor.

In the previous system of equations (1-3), it is noticed that the gravity gradient moment terms are included in the right hand where they weren't considered as disturbing or control torques. Moreover, the roll and yaw motions are coupled and must be solved simultaneously. While the pitch motion, which is given by eqn. 2 , is uncoupled with that of roll and yaw. So, the pitch attitude is controlled by a torque capabilities of a momentum wheel, $h_{w y o}$, which is set initially at some nominal biased value and it may be changed owing to the external disturbances acting about the $y_{b}$ axis however the other angular momentum components are assumed to be dumped [2] and [7] (Ouhocine et al., 2004 and Esmailzadeh et al., 2011).

\subsubsection{Laser Control Torque}

The control torque is considered to be that produced by a laser beam that was fired from a ground station towards the satellite. The components of laser torque in the ECI frame are given by [6] (Khalifa, 2012):

$$
\begin{aligned}
& T_{l x}=\frac{s}{c} \beta \cos \vartheta \int\left(R_{y} u_{z}-R_{z} u_{y}\right) d A \\
& T_{l y}=\frac{s}{c} \beta \cos \vartheta \int\left(R_{z} u_{x}-R_{x} u_{z}\right) d A \\
& T_{l z}=\frac{s}{c} \beta \cos \vartheta \int\left(R_{x} u_{y}-R_{y} u_{x}\right) d A
\end{aligned}
$$

the speed of light, $\beta$ is a function of thermo-optical properties of the satellite surface, $\vartheta$ is the angle between the incident unit vector $\hat{u}$ and the satellite surface normal and $\hat{R}$ is the unit vector directed from the spacecraft's center of mass to the element of satellite projected area $d A$. The unit vector $\hat{u}$ can be transformed to the body fixed frame as follows:

$$
\widehat{u}=A \hat{u}_{N}
$$

Where $A$ is the rotation matrix and $\hat{u}_{N}$ is the unite vector of laser beam incident direction in the ECI as shown in figure 2 . The components of the unit vector $\hat{u}_{N}$ are given by (NS. Khalifa, 2012):

$$
\begin{gathered}
u_{N x}=r(\cos \Omega \cos (\beta+v)-\sin \Omega \sin (\beta+v) \cos i)- \\
G_{1} \cos \varphi_{g} \cos \xi \\
u_{N y}=r(\sin \Omega \cos (\beta+v)+\cos \Omega \sin (\beta+v) \cos i)- \\
G_{1} \cos \varphi_{g} \sin \xi \\
u_{N z}=r \sin (\beta+v) \sin i-G_{2} \sin \varphi_{g}
\end{gathered}
$$

where $\Omega$ is longitude of the ascending node, $\beta$ is argument of perigee, $v$ is the true anomaly, $i$ is the inclination, $a$ is the semi-major axis and $e$ is the eccentricity.

$$
\begin{aligned}
G_{1} & =\frac{a_{e}}{\left(1-\left(2 f_{e}-f_{e}^{2}\right) \sin ^{2} \varphi_{g}\right)^{1 / 2}}+h \\
G_{2} & =\frac{a_{e}\left(1-f_{e}\right)^{2}}{\left(1-\left(2 f_{e}-f_{e}^{2}\right) \sin ^{2} \varphi_{g}\right)^{1 / 2}}+h
\end{aligned}
$$

where $a_{e}$ is the Earth's equatorial radius, $f_{e}^{\prime}$ is the Earth's flattening, $\varphi_{g}$ is the geodetic latitude, $h$ is the height above sea level and $\xi$ is the sidereal time.

where $s$ is the laser beam intensity at the satellite surface, $c$ is

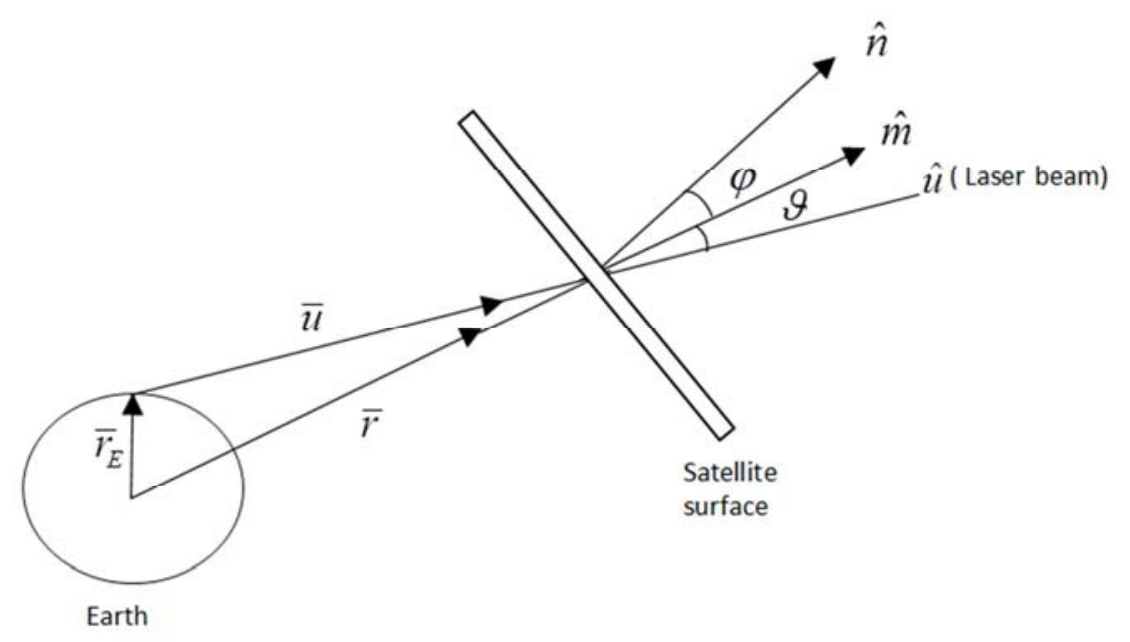

Figure 2. The laser intensity delivered to the satellite surface.

\section{System Simulation}

The model is implemented on a hypothetical $3 \mathrm{U}$ cubesat in a nearly circular LEO orbit. The simulated results were conducted using Matlab7 using some input assumptions, which are given in tables 1 and 2. The control configuration based only on measuring the response of roll and yaw angles via several runs 
for analyzing the passive and active control under the effect of gravity gradient and laser torques in different cases.

Table 1. The satellite orbit.

\begin{tabular}{ll}
\hline Orbit & Sun-synchronous \\
\hline Inclination & $98^{\circ}$ \\
Altitude & $600 \mathrm{Km}$ \\
Period & $5732 \mathrm{~s}$ \\
\hline
\end{tabular}

Table 2. The input parameters.

\begin{tabular}{ll}
\hline$\left\{\phi_{o}, \theta_{o}, \psi_{o}\right\}$ & $\left\{10^{\circ}, 0^{\circ}, 0^{\circ}\right\}$ \\
$\left\{I_{x}, I_{y}, I_{z}\right\}$ & $\{0.025,0.025,0.05) \mathrm{Kg} \mathrm{m}^{2}$ \\
$\left\{h_{w x}, h_{w y}, h_{w z}\right\}$ & $\{0,0.0035,0) \mathrm{Kg} \mathrm{m}^{2} / \mathrm{s}$ \\
$\omega_{o}$ & $0.0010764 \mathrm{rad} / \mathrm{s}$ \\
\hline
\end{tabular}

\subsection{Passive Control}

For passive control, it is assumed that there were no momentum devices and control torque. In this section two cases of passive control will be simulated; the purely passive control under gravity gradient torque only then in presence of laser torque.

\subsubsection{Purely Passive Gravity Gradient Attitude Control}

In this type of control, the other natural disturbances torques are ignored. Then the equations of roll and yaw motions (eqs. 1 and 3) will be reduced to the following form:

$$
\begin{aligned}
& I_{x} \ddot{\phi}+4 \omega_{o}^{2}\left(I_{y}-I_{z}\right) \phi+\omega_{o}\left(I_{y}-I_{z}-I_{x}\right) \dot{\psi}=0 \\
& I_{z} \ddot{\psi}+\omega_{o}\left(I_{z}+I_{x}-I_{y}\right) \dot{\phi}+\omega_{o}{ }^{2}\left(I_{y}-I_{x}\right) \psi=0
\end{aligned}
$$
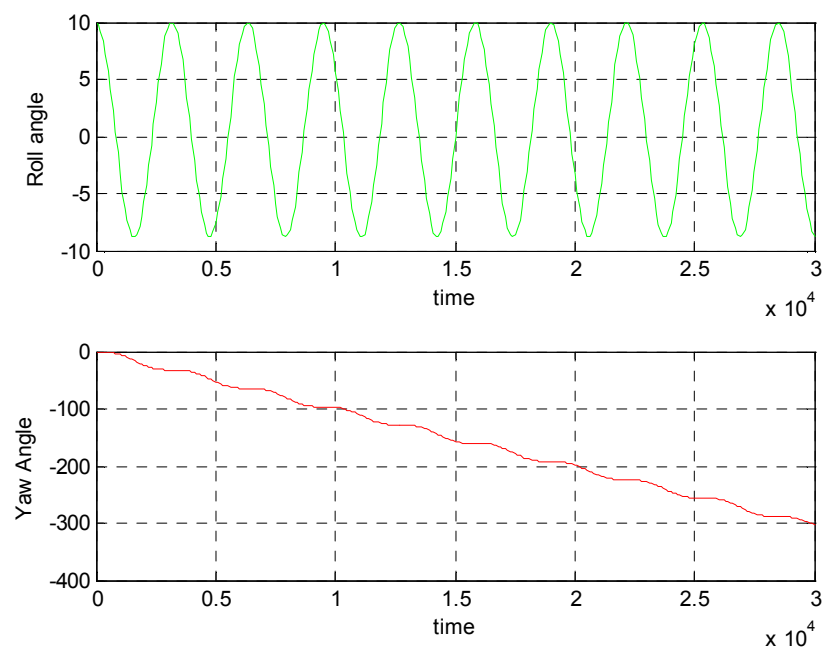

Figure 3. Response of roll and yaw angles using the passive attitude control.

The responses of the roll and yaw angles were illustrated in figure 3. The performance of roll angle shows that the motion is un-damped oscillation i. e. the satellite is marginally stable for roll angle. However, the yaw motion shows a divergent behavior.

\subsubsection{Laser Passive Attitude Control}

Having applied laser torque seeking for controlling the satellite behavior, the equations of roll and yaw motions in (eqs. 1 and 3) will be reduced to:

$$
\begin{aligned}
& I_{x} \ddot{\phi}+4 \omega_{o}^{2}\left(I_{y}-I_{z}\right) \phi+\omega_{o}\left(I_{y}-I_{z}-I_{x}\right) \dot{\psi}=T_{l x} \\
& I_{z} \ddot{\psi}+\omega_{o}\left(I_{z}+I_{x}-I_{y}\right) \dot{\phi}+\omega_{o}^{2}\left(I_{y}-I_{x}\right) \psi=T_{l z}
\end{aligned}
$$

For the purpose of this analysis, the following postulates are applied:

1. The radiation fall normal to the satellite surface.

2. The satellite surface is considered to be perfectly reflecting surface. So, the laser force will be duplicated.

3. (3-2-1) Euler rotation is assumed with $\psi$ being the first angle of rotation, $\theta$ being the angle of the second rotation and $\varphi$ being the angle of the third rotation.

Based on the previous postulates and applying a laser torque of about $10 \mathrm{kw}$ power, the components of laser torque are given by:

$$
\begin{aligned}
& T_{l x}= \cos \theta\left(-4.1 \times 10^{-6} \cos \phi+1.8 \times 10^{-5} \sin \phi\right)+ \\
&+ \cos \phi\left(\cos \psi\left(1.4 \times 10^{-6}+3.9 \times 10^{-6} \sin \theta\right)\right. \\
&+\left.\left(1.6-3.4 \times 10^{-7} \sin \theta\right) \sin \psi\right) \\
&+\sin \phi\left(\cos \psi\left(3.4 \times 10^{-7}-1.6 \times 10^{-5} \sin \theta\right)\right. \\
&\left.+\left(3.9 \times 10^{-6}+1.4 \times 10^{-6} \sin \theta\right) \sin \psi\right) \\
& T_{l z}=\cos \phi\left(-3.4 \times 10^{-7} \cos \psi-3.9 \times 10^{-6} \sin \psi\right)+ \\
&+\cos \theta\left(-3.9 \times 10^{-6} \cos \psi-4.1 \times 10^{-6} \sin \phi+3.4\right. \\
&\left.\times 10^{-7} \sin \psi\right)+ \\
&+\sin \theta\left(-4.1 \times 10^{-6}+3.9 \times 10^{-6} \cos \psi \sin \phi-3.4\right. \\
&\left.\times 10^{-7} \sin \phi \sin \psi\right)
\end{aligned}
$$

The responses of the roll and yaw angles using different magnitude of laser torque as passive control in addition to the gravity gradient are illustrated in figure 4.
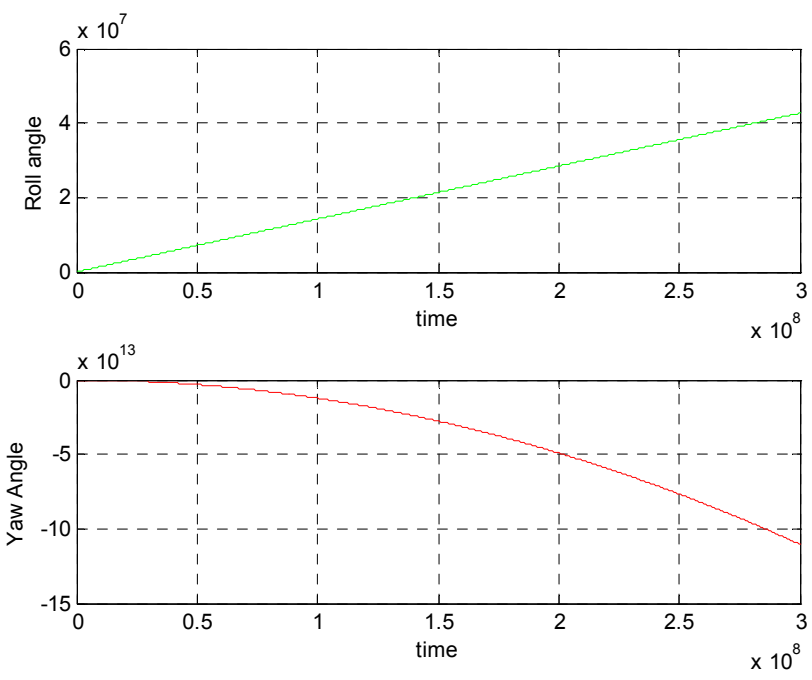

using $25 \mathrm{kw}$ laser 

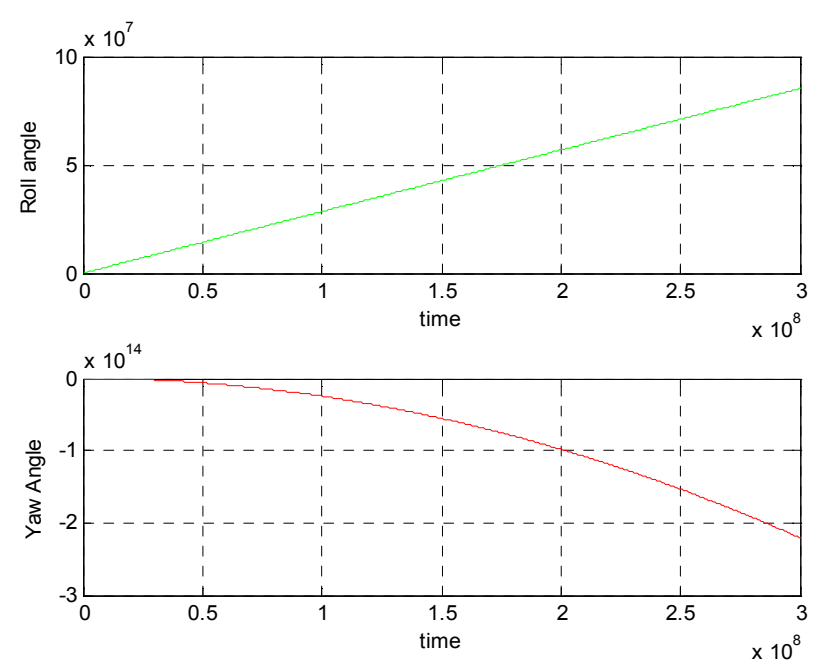

using $50 \mathrm{kw}$ laser
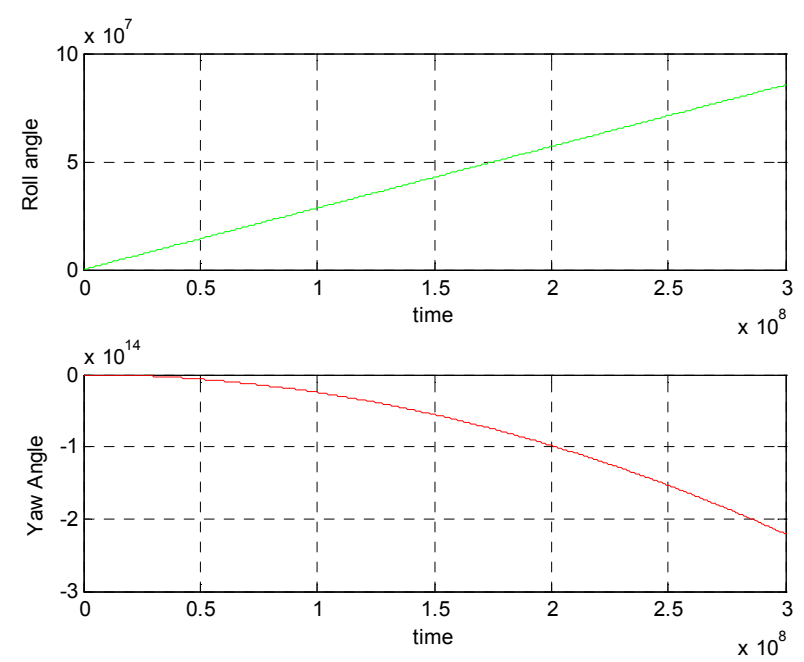

using $75 \mathrm{kw}$ laser
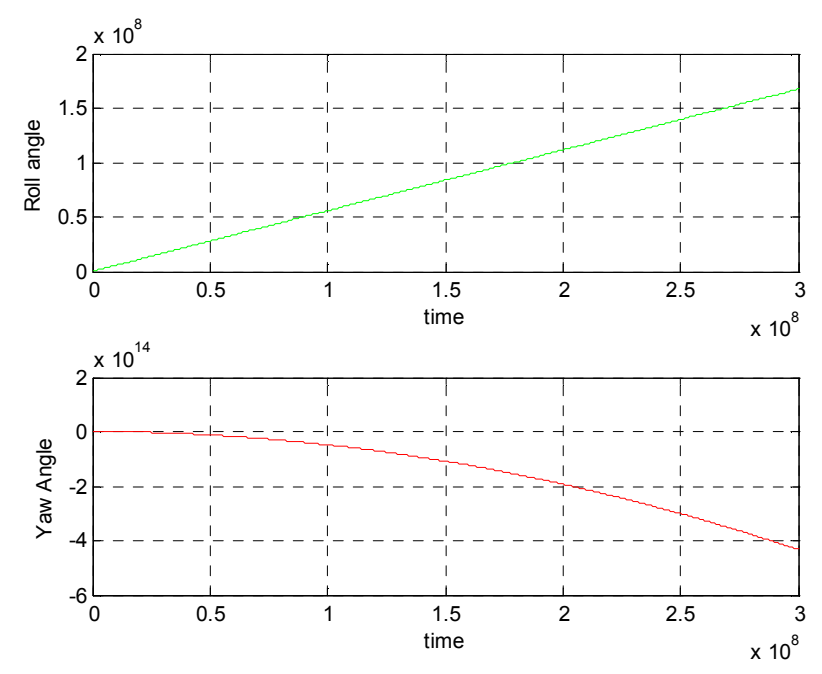

using $75 \mathrm{kw}$ laser

Figure 4. Response of roll and yaw angles using the passive attitude control under the effect of different magnitude of laser torque.
The obtained results, Fig. 4, show a divergent behavior for both roll and yaw motions under the effect of different laser torque magnitudes. Therefore, attitude stability cannot be achieved by using passive laser control in existence of gravity gradient only. Then introducing of damping control is considered to be necessary. In the next subsection, a study of active control based on PD controller, moreover the effect of laser torque on the system performance will be explained.

\subsection{Active Control}

Active control technique is commonly used for damping. The basic idea dominating any damping system is to afford additional dynamic elements within a system, and to provide suitable coupling between the system variables in order to obtaining largest possible damping of satellite angular motion. Consequently, it is assumed that the satellite was equipped with a momentum wheel to provide an inertial stability about the pitch axis. The wheel angular momentum components are given in table 2 .

\section{Controller Design}

In this work, the implemented control algorithm here is PD controller. In comparison to the other alternatives control algorithm, the Proportional Derivative (PD) controller is chosen because it can be designed using classical control theory and therefore gain selection is relatively intuitive. Moreover, the PD controller does not depend on linear equations of motion and therefore is capable of large angle manoeuvres by incorporating the nonlinear dynamics (Francois-Lavet, 2010).

The control command equation is given by:

$$
\begin{aligned}
& T_{c x}=k_{1} \phi+k_{2} \dot{\phi} \\
& T_{c z}=k_{1} \psi+k_{2} \dot{\psi}
\end{aligned}
$$

Where $k_{1}$ and $k_{2}$ are the proportional gains which are selected by proper tune in order to achieve desired response. The proportional and derivative gains are given in Table 3:

Table 3. The parameters of PD controller.

\begin{tabular}{ll}
\hline$k_{1}$ & $0.6 \times 10^{-5}$ \\
$k_{2}$ & $44.5 \times 10^{-5}$ \\
\hline
\end{tabular}

The simulation for active control will be conducted first for PD and momentum wheel, and then the effect of laser torque will be considered.

\subsection{PD Controller for a Momentum Biased Cubesat Without Laser Torque}

Assuming that one momentum wheel is added to the satellite equipment to provide inertial stability about the pitch axis. The gains of PD controller are tuned manually to optimize the responses of the roll and yaw angles. 

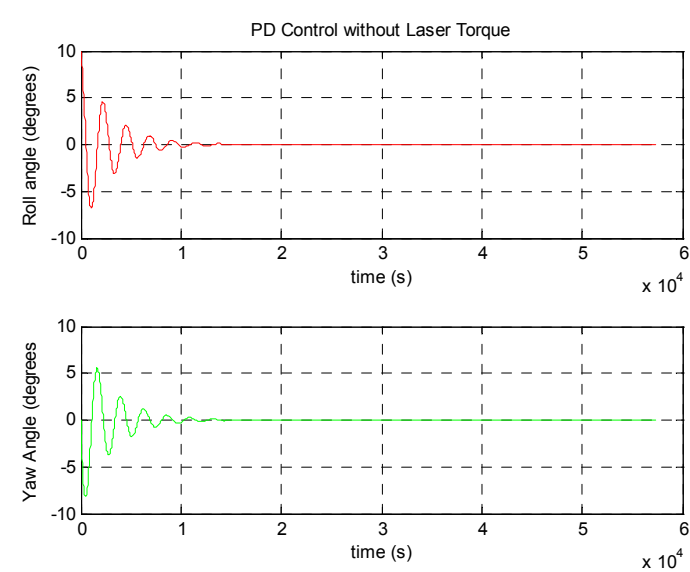

Figure 5. Responses of yaw and roll using PD controller for momentum biased cubesat.

It observed from Fig. 5 that the stability of the CubeSat is assured. The results show that response in roll and yaw angles starting from the initial position (as in Table 2), are able to converge to zero in about two orbit time. The steady state error is zero in both roll and yaw.

\subsection{PD Controller with Laser Effect for a Momentum Biased Cubesat}

The simulation is carried out using different magnitudes of laser torque which are as given in table 3 . The responses of the roll and yaw angles using different magnitude of laser torque using PD controller were illustrated in figure 6.
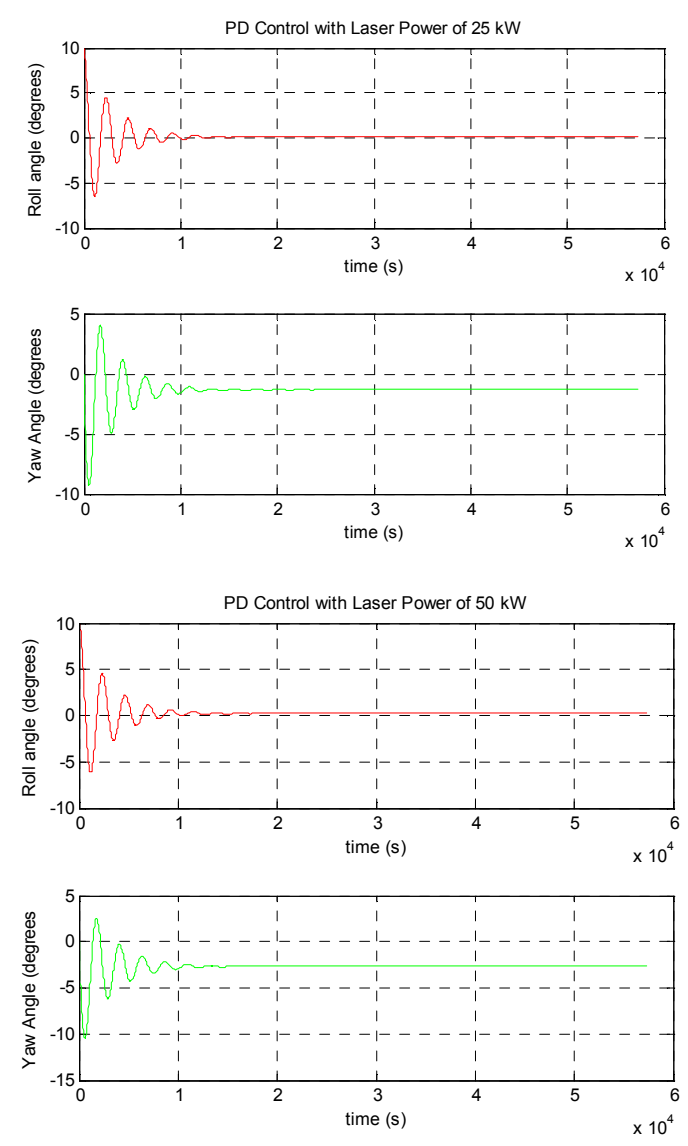
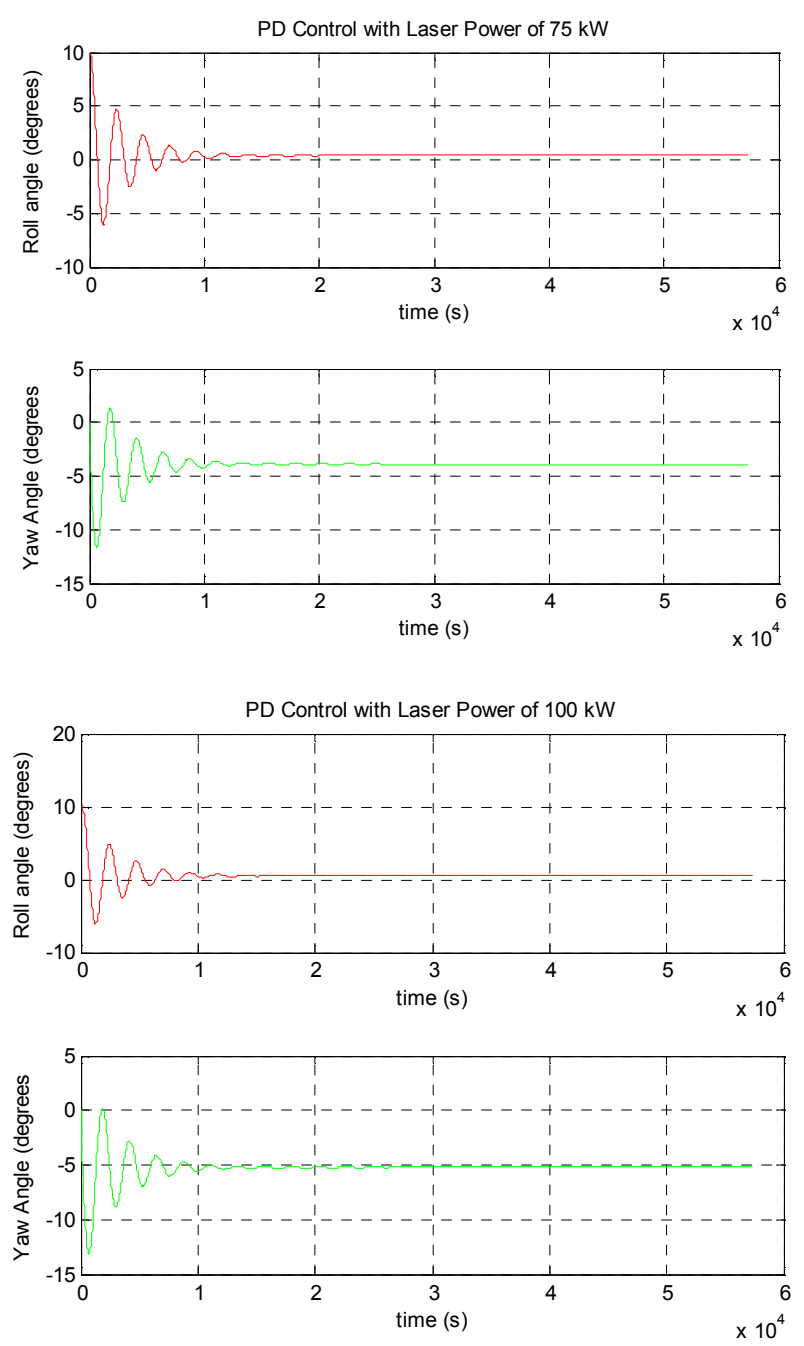

Figure 6. Response of yaw and roll using $P D$ controller with different magnitude of laser torque.

It can be shown in Fig. 6 that the effect of laser torque can be summarized as follows:

1. The laser torque increases the steady state errors in both roll and yaw angles. It is obvious it has more significant effect in yaw angle than rol angle; this is expectedly due to larger negative component of laser torque in $\mathrm{z}$-axis than $\mathrm{x}$-axis.

2. Having increased the magnitude of laser torque, the steady state errors in both axes are increased.

3. It is noted that the laser torque has no effect on system stability or the time response of system.

\section{Conclusion}

For this simple case of study, authors investigated and assessed the effects of ground based laser torque with different magnitudes on both passive and active control. The obtained results illustrated that the satellite stability cannot be realized on using gravity gradient torque and laser torque for passive control.

However with PD active control biased on momentum wheel, the results show that the stability is assured and laser 
torque has no effect on both system stability and system time response.

Increasing

The laser torque magnitudes have the consequences of increasing the steady state errors in the response of roll and yaw angles. The effect of laser torque is more significant in yaw angle response than roll angle response.

Future work

The authors aim to:

1. Study system stability under the effect of laser torque.

2. Study laser active control by replacing the linear PD controller with the variable structure control algorithm.

3. Study the problem in presence of different disturbing torques (e. g. aerodynamics, solar radiation, etc), more than one reaction wheel and magnetic torquer.

4. Study the problem in the quaternion frame.

\section{References}

[1] Abdualhamitbilal and E. M. Jafarov, 2006, "Performances comparison of linear and sliding mode attitude controllers for flexible spacecraft with reaction wheels, proceedings of the 2006 international workshop on variable structure system. Alghero, Italy, 2006.

[2] C. Ouhocine, M. N. Filipski, S. B. Mohd Noor and M. R. Ajir,
"Small Satellite Attitude Control and simulation", jurnal Mekanikal, Bil.17, 2004.

[3] J. C. Hsu, Y. S. Lim and A. U. Meyer, "on active control of satyellites", IEEE Transactions on Military Electronics, 1965.

[4] K. Vega, "Attitude Control System for CubeSat for Ions, Neutrals, Electrons and MAGnetic Field (CINEMA)", MSc. Thesis, UNIVERSITY OF CALIFORNIA, BERKELEY, 2009.

[5] M. J. Sidi, "Spacecraft Dynamics", Cambridge University Press, 1997.

[6] N. S. khalifa, "Effect of an Artificial Radiant Force on the Spacecraft's Orbit”, Phd. Thesis, Cairo university, 2009.

[7] R. Esmailzadeh, H. Arefkhani and S. Davoodi, "Active control and attitude stabilization of a momentum-biased satellite without yaw measurements, IEEE, Electrical Engineering (ICEE), 19th Iranian Conference, 2011.

[8] Samir Rawashdeh" PASSIVE ATTITUDE STABILIZATION FOR SMALL SATELLITES", Msc. Thesis, University of Kentucky, Kentucky, 2009.

[9] Francois-Lavet, "Study of passive and active attitude control systems for the OUFTI nanosatellites", Msc. Thesis, University of Liège, 2010.

[10] Wertz J. R. editor," Spacecraft Attitude Determination and Control", D. Reidel, Dordrecht, Holland, 1978. 